\title{
Stage IIIB Small Intestinal Gastrointestinal Stromal Tumor
}

National Cancer Institute

\section{Source}

National Cancer Institute. Stage IIIB Small Intestinal Gastrointestinal Stromal Tumor. NCI

Thesaurus. Code C87834.

Stage IIIB includes: (T2, NO, MO, Mitotic rate: High); (T3, NO, MO, Mitotic rate: High); (T4, NO, MO, Mitotic rate: High). T2: Tumor more than $2 \mathrm{~cm}$, but not more than $5 \mathrm{~cm}$, in greatest dimension. T3: T umor more than $5 \mathrm{~cm}$, but not more than $10 \mathrm{~cm}$, in greatest dimension. T4: Tumor more than $10 \mathrm{~cm}$ in greatest dimension. N0: No regional lymph node metastasis. M0: No distant metastasis. (AJCC 7th ed.) 